\title{
Comparação das Respostas Fisiológicas \\ e Perceptuais Obtidas Durante \\ Caminhada na Esteira em Ritmo \\ Autosselecionado Entre os Sexos
}

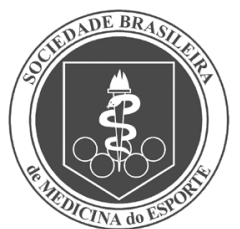

Artigo Original

\author{
Physiological and Perception Responses Comparison During Treadmill \\ Walking at Self-Selected Pace Between Genders
}

\begin{abstract}
Kleverton Krinski
Hassan M. Elsangedy ${ }^{1}$

Cosme F. Buzzachera²

Heriberto Colombo'

Ragami Chaves Alves ${ }^{1}$

Bruno V. Santos'

Maressa Priscila Krause

Laura Guidetti ${ }^{2}$

Carlo Baldari ${ }^{2}$

Sergio G. Dasilva'

1. Departamento de Educação Física da Universidade Federal do Paraná, Curitiba, Brasil.

2. Universidade de Estudos de Roma

"Foro Italico", Roma, Itália.
\end{abstract}

\section{Endereço para correspondência:}

Sergio Gregorio da Silva, Ph.D.

Rua Coração de Maria, 92 -

Jd. Botânico

80215-370 - Curitiba, Paraná - Brasil

E-mail: sergiogregorio@ufpr.br

\section{RESUMO}

O objetivo do presente estudo foi comparar as respostas fisiológicas e perceptuais entre os sexos durante a caminhada na esteira em ritmo autosselecionado. Participaram 17 homens e 17 mulheres, fisicamente ativos, com média de idade de 23,32 \pm 3,06 anos, submetidos a duas sessões experimentais: (I) avaliação antropométrica e teste incremental máximo, e (II) um teste de 20 minutos de caminhada na esteira em ritmo autosselecionado. Para a análise estatística, empregou-se teste $t$ de Student para medidas independentes no intuito de verificar as possíveis diferenças entre os sexos, adotando $p \leq 0,05$. A velocidade de caminhada autosselecionada pela amostra masculina foi superior à verificada na feminina $(1,65$ $\pm 0,18$ e 1,50 $\pm 0,12 \mathrm{~m} \cdot \mathrm{seg}^{-1}$, respectivamente) o que consequentemente resultou em maior $\dot{\mathrm{V}} \mathrm{O}_{2}$ absoluto nos homens comparado às mulheres (21,2 $\pm 5,5$ e 18,3 $\pm 2,7$, respectivamente). No entanto, ambos os sexos buscaram caminhar em mesma intensidade relativa \% $\dot{V}_{2 \text { máx }}(37,5 \pm 10,7$ homens e 40,3 \pm 7,2 mulheres). Em relação à percepção subjetiva de esforço (PSE), podemos verificar que ambos os sexos não demonstraram diferenças significativas (10,2 $\pm 1,0$ homens e 9,8 $\pm 1,2$ mulheres). Os achados do presente estudo demonstram que, independente do sexo, jovens adultos fisicamente ativos autosselecionaram similar intensidade relativa que refletiu em similar PSE. Além disso, a caminhada em intensidade autosselecionada demonstra-se como estímulo insuficiente para proporcionar melhora no condicionamento cardiorrespiratório nesta população.

Palavras-chave: consumo de oxigênio, frequência cardíaca, esforço, atividade física.

\section{ABSTRACT}

The aim of this study was to compare physiological and perception responses between genders during treadmill walking at self-selected pace. 17 men and 17 women aged $23.32 \pm 3.06 \mathrm{yr}$ were investigated: (I) anthropometric assessment and incremental exhaustion test, (II) a 20-minute walking bout on treadmill at their self-selected pace. The independent $t$ test was utilized to verify any gender differences, with a level of $p<0.05$. The self-selected walking pace was significantly greater in men than women (1.65 \pm 0.18 and $1.50 \pm 0.12 \mathrm{~m} \cdot \mathrm{sec}^{-1}$, respectively), and consequently resulted in higher absolute $\mathrm{VO}_{2}$ for men compared to women $(21.2 \pm 5.5$ and $18.3 \pm 2.7$, respectively). However, both genders self-selected a similar relative exercise intensity $\% \mathrm{VO}_{2 \max }(37.5 \pm 10.7$ and $40.3 \pm 7.2$ for men and women, respectively). The subjective perceived exertion (SPE) did not differ between genders (10.2 \pm 1.0 and $9.8 \pm 1.2$ for men and women, respectively). The results of the present study demonstrated that regardless of gender, physically active young adults self-selected a similar relative exercise intensity that reflected in similar SPE. Furthermore, gait at self-selected intensity was insufficient to improve cardiorespiratory fitness in this sample.

Keywords: oxygen uptake, heart rate, exertion, physical activity. 


\section{INTRODUÇÃO}

A caminhada é uma forma conveniente e popular de exercício físico executada por homens e mulheres, devido à sua segurança, efetividade e simplicidade ${ }^{(1,2)}$, podendo trazer benefícios à saúde e melhora do condicionamento cardiorrespiratório ${ }^{(3)}$, quando realizada em quantidade e intensidade apropriadas ${ }^{(2)}$. O Colégio Americano de Medicina do Esporte $(A C S M)^{(2)}$, recomenda um mínimo de 30 minutos de atividade física durante a maioria, se não todos os dias da semana, a uma intensidade correspondente a 50-85\% do consumo máximo de oxigênio $\left(\mathrm{VO}_{2 \text { Máx }}\right)$ e $55-90 \%$ da frequência cardíaca máxima $\left(\mathrm{FC}_{\text {Máx }}\right)$ necessárias para melhoria da aptidão cardiorrespiratória $(A C R)^{(2)}$.

Contudo, apesar da importância de estímulo fisiológico adequado durante o exercício ser indiscutível para obter modificações orgânicas benéficas, Dishman et al.(1) observaram que a maioria dos sujeitos engajados em programas de atividade física buscam exercitar-se em intensidade autosselecionada em detrimento da previamente prescrita. De acordo com Johnson e Phipps ${ }^{(4)}$, os indivíduos buscam ajustar a intensidade que selecionam durante o exercício através do uso da taxa de percepção subjetiva de esforço (PSE), a qual pode ser mensurada mediante a utilização da escala de Borg (6-20), demonstrando ampla aplicabilidade e facilidade, dispensando equipamentos específicos ou extensiva experiência por parte dos sujeitos, permitindo quantificar os sinais do sistema neuromuscular e cardiorrespiratório durante o exercício(5).

Previas investigações ${ }^{(6,7)}$ encontraram resultados controversos para a PSE entre os sexos durante o exercício, reportando não haver diferenças( ${ }^{(6)}$, bem como percepção de esforço mais elevada em mulheres comparado a homens ${ }^{(7)}$. Entretanto, a maioria dos estudos avaliando as respostas da PSE entre os sexos durante o exercício foram realizadas com protocolos de intensidade preestabelecidos ${ }^{(5)}$, em atividades como corridas $^{(8)}$ e exercício realizado em cicloergômetro ${ }^{(6)}$, com poucas informações referentes às respostas fisiológicas e perceptuais durante o exercício em intensidades autosselecionadas. Neste sentido, o presente estudo teve como objetivo avaliar as respostas fisiológicas e perceptuais entre os sexos durante caminhada realizada na esteira em intensidade autosselecionada.

\section{MÉTODOS}

\section{Sujeitos}

A amostra foi composta de 34 adultos (17 homens e 17 mulheres, com média de idade de 23,32 \pm 3,06 anos) fisicamente ativos, com experiência em caminhada realizada na esteira. Ambos os sexos foram submetidas a avaliação médica inicial no intuito de excluir indivíduos que apresentassem problemas metabólicos, cardiovasculares, osteomioarticulares e outras condições que influenciassem ou restrigissem a aplicação de um teste de esforço.

Para determinar o número de sujeitos, foi utilizado cálculo amostral adotando poder de 0,80, alfa de 0,05 e magnitude de efeito de 1,17 , estimando 17 sujeitos para cada grupo experimental no modelo que verifica as diferenças nas variáveis analisadas entre os dois grupos (amostras independentes); desta forma, o número de sujeitos foi suficiente para testar as hipóteses a um alfa de 0,05.

Os sujeitos foram informados sobre os procedimentos utilizados, possíveis benefícios e riscos atrelados, conforme as diretrizes propostas na resolução 196/96 e aprovado pelo Comitê de Ética em Pesquisa da Universidade Federal do Paraná. Os participantes foram instruídos a não realizar atividade física vigorosa nas 24 horas anteriores aos testes, bem como não ingerirem qualquer tipo de alimento e bebidas cafeinadas por um período de duas horas antecedentes ao seu início. A média de temperatura ao longo dos testes foi de $20^{\circ} \mathrm{C}$ a $22^{\circ} \mathrm{C}$ e umidade relativa entre 50 e $60 \%{ }^{(2)}$.

\section{Sessão de familiarização}

Na sessão de familiarização os sujeitos assinaram o Termo de Consentimento Livre e Esclarecido, seguido de avaliação antropométrica e familiarização com os aparatos do analisador de gases. Na sequência, foram explanadas instruções padronizadas referentes à escala de Borg (6-20), e esclarecido o procedimento para ajuste da velocidade através dos sensores acoplados à esteira, e testado pelos participantes na execução de 10 minutos de caminhada em ritmo autosselecionado.

\section{Teste incremental}

Os participantes realizaram aquecimento em esteira (marca Reebok Fitness, modelo X-fit 7, Londres, Reino Unido) de cinco minutos a uma velocidade padrão de $4,0 \mathrm{~km} / \mathrm{h}$ sem inclinação, seguido de teste incremental até exaustão em esteira conduzido mediante utilização de protocolo de Bruce ${ }^{(9)}$. O teste foi finalizado no ponto de exaustão volitiva dos participantes, os quais foram encorajados verbalmente a se manterem em exercício pelo maior tempo possível. A determinação dos parâmetros fisiológicos $\left(\mathrm{FC} \mathrm{eVO}_{2}\right)$ foi realizada através de analisador de gases portátil coletando os dados respiração a respiração (breath by breath), e os parâmetros perceptuais a cada minuto.

\section{Teste de caminhada em esteira de $\mathbf{2 0}$ minutos}

$O$ teste de 20 minutos de caminhada na esteira em ritmo autosselecionado foi conduzido após cinco minutos de aquecimento inicial em velocidade de 4,0km/h sem inclinação. Durante a realização do teste, os participantes foram instruídos a ajustar um ritmo autosselecionado, conforme os procedimentos propostos por Dishman et al.(1) mediante a utilização de sensores de controle de velocidade acoplados à esteira. Este ajuste foi realizado ad libitum durante o primeiro minuto de caminhada e, posteriormente, somente nos minutos 00:05, 00:10 e 00:15. Contudo, o marcador de velocidade foi ocultado para o avaliado não ser influenciado na autosseleção do ritmo de caminhada. Novamente, a determinação dos parâmetros fisiológicos foi realizada a cada 15 segundos utilizando analisador de gases portátil coletando dados respiração a respiração, e os parâmetros perceptuais a cada minuto.

\section{Percepção subjetiva de esforço (PSE)}

A PSE foi determinada através da escala de Borg (6-20)(2); esse instrumento é composto de uma escala Likert de 15 pontos, variando de 6 a 20, iniciando em "muito, muito leve" e finalizando em "muito, muito difícil". A ancoragem da escala de Borg (6-20) foi realizada mediante teste incremental máximo em esteira a fim de padronizar a baixa e alta percepção.

\section{Medidas antropométricas}

As variáveis antropométricas massa corporal (MC, em kg; balança marca Toledo, modelo 2096), estatura (EST, em cm; estadiômetro marca Sanny, modelo Standard) e índice de massa corporal (IMC, em $\mathrm{kg} / \mathrm{m}^{2}$ ) foram obtidos conforme os procedimentos propostos por Gordon et al. ${ }^{(10)}$. A densidade corporal foi mensurada pelo método de espessura de dobras cutâneas, de acordo com a equação proposta por Durnin e Womersley ${ }^{(11)}$. Posteriormente, o percentual de gordura corporal (\% gordura) foi obtido mediante a equação de Siri(12).

\section{Respostas fisiológicas}

$\mathrm{O}$ consumo de oxigênio $\left(\mathrm{VO}_{2}\right)$ foi registrado a cada respiração, determinado através de um sistema portátil de espirometria computadorizado de circuito aberto (marca Cosmed K4b2, Roma, Itália). Antes de cada teste, os sistemas de análise do $\mathrm{O}_{2}$ e $\mathrm{CO}_{2}$ foram calibrados usando o ar ambiente e um gás com concentrações conhecidas de $\mathrm{O}_{2}$ e $\mathrm{CO}_{2}$, enquanto a turbina bidirecional foi calibrada usando seringa de 3-L (marca Hans Rudolph, modelo 5530, Kansas City, Missouri, EUA). 
Os dados dos testes foram reduzidos a médias com intervalos de 15 segundos. Para a determinação do $\mathrm{VO}_{2 \text { Máx }}$ dois critérios deveriam ser atingidos: (a) platô do $\mathrm{VO}_{2}$, indicado por uma diferença de $<2,1 \mathrm{ml} / \mathrm{kg} /$ min entre os dois últimos estágios do teste; (b) taxas de troca respiratória (RER) > 1,10; e (c) frequência cardíaca dentro de $\pm 10 \mathrm{bpm}$ para cada sujeito através do valor máximo predito pela idade ${ }^{(13)}$. O limiar ventilatório (LV) foi calculado individualmente conforme os procedimentos estabelecidos por Caiozzo et al.(13), e visualmente identificado como o ponto em que a plotagem da razão ventilação minuto por consumo de oxigênio $\left(\mathrm{VENO}_{2}\right)$ versus a razão ventilação minuto por produção de $\mathrm{CO}_{2}$ (VENCO2) desvia da normalidade.

A FC foi obtida através de fita elástica ajustada ao tórax e transmissor de rádio ligado ao sistema de análise metabólica computadorizada (Polar Oy model Sports Tester, Kempele, Finland). A frequência cardíaca máxima $\left(F C_{\text {Máx }}\right.$ e frequência cardíaca no limiar ventilatório $\left(F C_{L V}\right)$ foram determinadas como a maior $F C$ média (intervalo de um minuto) verificada no último estágio completo do teste incremental e no limiar ventilatório.

\section{Procedimentos estatísticos}

Medidas de tendência central e variabilidade foram utilizadas. Para testar a normalidade dos dados utilizou-se o teste de KolmogorovSmirnov. As diferenças entre os sexos para as variáveis fisiológicas foram determinadas pelo teste $t$ de Student para amostras independentes adotando nível de significância de $p \leq 0,05$. A magnitude de efeito (d) foi reportada para cada comparação determinada através dos procedimentos propostos por Cohen ${ }^{(14)}$. Os procedimentos estatísticos foram realizados mediante o Statistical Package for the Social Sciences (SPSS, versão 13.0) for Windows.

\section{RESULTADOS}

As características dos sujeitos são apresentadas na tabela 1, demonstrando MC e estatura superiores nos homens $(P<0,05)$ comparados às mulheres.

Tabela 1. Características descritivas dos participantes.

\begin{tabular}{c|c|c|c|c|c|c|c|c}
\hline & \multicolumn{2}{|c|}{ Geral } & \multicolumn{2}{|c|}{ Homens } & \multicolumn{2}{c|}{ Mulheres } & \multirow{2}{*}{$p$} & \multirow{2}{*}{$\boldsymbol{d}$} \\
& Média & DP & Média & DP & Média & DP & & \\
\hline Idade & 23,32 & 3,06 & 24,05 & 3,32 & 22,58 & 2,67 & 0,16 & 0,50 \\
\hline MC & 65,39 & 10,75 & 71,96 & 10,15 & 58,81 & 6,59 & $0,01^{*}$ & 1,58 \\
\hline Estatura & 1,68 & 0,09 & 1,75 & 0,06 & 1,62 & 0,06 & $0,01^{*}$ & 2,23 \\
\hline IMC & 22,77 & 2,10 & 23,31 & 2,21 & 22,23 & 1,89 & 0,13 & 0,54 \\
\hline \% gordura & 19,67 & 3,57 & 18,38 & 3,20 & 20,88 & 3,55 & 0,42 & 0,76 \\
\hline
\end{tabular}

Dados em média \pm DP. MC: massa coporal; IMC: índice de massa corporal; \% gordura: perceptual de gordura . * diferença estatisticamente significativa $(p \leq 0,05)$ entre homens e mulheres.

As respostas fisiológicas e perceptuais obtidas durante o teste incremental até a exaustão estão apresentadas na tabela 2 . 0 teste $t$ de Student para amostras independentes revelou que foram encontradas diferenças para o $\dot{\mathrm{V}} \mathrm{O}_{2 \text { Máx }}(\mathrm{P}<0,01)$, e $\dot{\mathrm{V}} \mathrm{E}_{\text {Max }}(\mathrm{P}<0,01)$, sendo maior entre os homens.

Os parâmetros fisiológicos e perceptuais obtidos durante o teste de 20 minutos de caminhada em ritmo autosselecionado estão apresentados na tabela 3.

Podem-se observar diferenças estatisticamente significantes entre os sexos para o $\dot{\mathrm{V}} \mathrm{O}_{2}$ absoluto $(\mathrm{P} \leq 0,05)$, e velocidade de caminhada $(P<0,01)$ durante o teste de 20 minutos em ritmo autosselecionado. Entretanto, não foram verificadas diferenças estatisticamente significantes para as respostas perceptuais.
Tabela 2. Respostas fisiológicas e perceptuais durante o teste incremental.

\begin{tabular}{|c|c|c|c|c|c|c|c|c|}
\hline & \multicolumn{2}{|c|}{$\begin{array}{c}\text { Geral } \\
(n=34)\end{array}$} & \multicolumn{2}{|c|}{$\begin{array}{l}\text { Homens } \\
(n=17)\end{array}$} & \multicolumn{2}{|c|}{$\begin{array}{c}\text { Mulheres } \\
(n=17)\end{array}$} & \multirow{2}{*}{$\begin{array}{c}\mathbf{P} \\
0,01^{*} \\
\end{array}$} & \multirow{2}{*}{$\begin{array}{c}d \\
2,04 \\
\end{array}$} \\
\hline$\dot{\mathrm{V}} \mathrm{O}_{2 \text { máx }}$ & 51,6 & 8,1 & 57,3 & 5,9 & 45,9 & 5,6 & & \\
\hline $\mathrm{FC}_{\text {máx }}$ & 190,0 & 6,1 & 189,8 & 5,4 & 190,1 & 7,0 & 0,91 & 0,04 \\
\hline VE & 122,0 & 29,1 & 143,1 & 26,5 & 100,9 & 10,1 & $0,01^{*}$ & 2,17 \\
\hline RER & 1,13 & 0,09 & 1,13 & 0,08 & 1,13 & 0,10 & 0,98 & 0,00 \\
\hline $\operatorname{PSE}_{\mathrm{LV}}(6-20)$ & 11,94 & 1,68 & 11,7 & 1,8 & 12,1 & 1,5 & 0,28 & 0,25 \\
\hline
\end{tabular}

Dados em média $\pm \mathrm{DP}$. $\dot{V}_{2 \text { max: }}$ : Consumo máximo de oxigênio; $\mathrm{FC}_{\text {max }}$ : frequência cardíaca máxima; VE: ventilaçăo pulmonar; RER: razão de troca respiratória PSE: percepção subjetiva de esforço no limiar ventilatório. * diferença estatisticamente significativa $(p \leq 0,05)$ entre homens e mulheres.

Tabela 3. Respostas fisiológicas e perceptuais durante o teste de caminhada de 20 minutos em intensidade autosselecionada.

\begin{tabular}{|c|c|c|c|c|c|c|c|c|}
\hline & \multicolumn{2}{|c|}{$\begin{array}{c}\text { Geral } \\
(n=34)\end{array}$} & \multicolumn{2}{|c|}{$\begin{array}{l}\text { Homen } \\
(n=17)\end{array}$} & \multicolumn{2}{|c|}{$\begin{array}{l}\text { Mulher } \\
(n=17)\end{array}$} & \multirow{2}{*}{$\begin{array}{c}\mathbf{P} \\
0,05^{*}\end{array}$} & \multirow{2}{*}{$\begin{array}{c}d \\
0,70\end{array}$} \\
\hline$\dot{\mathrm{V}} \mathrm{O}_{2}$ & 19,79 & 4,53 & 21,27 & 5,52 & 18,30 & 2,70 & & \\
\hline$\% \dot{\mathrm{V}} \mathrm{O}_{2 \text { máx }}$ & 38,94 & 9,15 & 37,54 & 10,75 & 40,34 & 7,27 & 0,38 & 0,31 \\
\hline$\% \dot{\mathrm{V}} \mathrm{O}_{2 \mathrm{LV}}$ & 54,9 & 15,0 & 55,0 & 18,3 & 54,8 & 11,3 & 0,96 & 0,01 \\
\hline $\mathrm{FC}(\mathrm{bpm})$ & 114,4 & 14,7 & 110,7 & 16,5 & 118,1 & 12,2 & 0,15 & 0,52 \\
\hline$\% \mathrm{FC}_{\max }$ & 60,25 & 7,65 & 58,38 & 8,86 & 62,12 & 5,91 & 0,15 & 0,51 \\
\hline$\% \mathrm{FC}_{V T}$ & 72,8 & 11,8 & 72,2 & 14,5 & 73,4 & 8,7 & 0,78 & 0,10 \\
\hline $\operatorname{PSE}(6-20)$ & 10,04 & 1,13 & 10,26 & 1,02 & 9,82 & 1,22 & 0,26 & 0,40 \\
\hline Vel $\left(m \cdot \operatorname{seg}^{-1}\right)$ & 1,58 & 0,17 & 1,65 & 0,18 & 1,50 & 0,12 & $0,01^{*}$ & 1,01 \\
\hline
\end{tabular}

Dados em média \pm DP. $\dot{V O}_{2}$ : consumo máximo de oxigênio; $\% \dot{V O}_{2 \text { max: }}$ percentual do consumo máximo de oxigênio massa coporal: \%VंO percentual do consumo máximo de oxigênio no limiar ventilatório: \% FC . percentua da frequência cardíca máxima: \%FC : percentual da frequência cardíaca máxima no limiar ventilatório: PSE: percepçăo subjetiva de esforço; Vel: velocidade em metros por segundo * diferença estatisticamente significativa $(p \leq 0,05)$ entre homens e mulheres.

\section{DISCUSSÃO}

A literatura contém ampla informação sobre as respostas fisiológicas e perceptuais entre os sexos durante o exercício; entretanto, esta relação é pouco conhecida em atividades de fácil acesso, populares e seguras como a caminhada desenvolvida em ritmo autosselecionado. Neste sentido, a presente investigação teve por objetivo buscar subsídios para a melhor compreensão das respostas fisiológicas e perceptuais obtidas durante a caminhada em ritmo autosselecionado na esteira entre os sexos.

Os resultados apresentados durante o teste de caminhada de 20 minutos demonstram que os homens autosselecionaram velocidade $\left(\mathrm{m} \cdot \mathrm{seg}^{-1}\right)$ superior a das mulheres $(1,65 \pm 0,18$ e 1,50 \pm 0,12, respectivamente). Estes resultados são similares aos encontrados por Finley e Cody ${ }^{(15)}$, que demonstraram que homens preferem caminhar a uma velocidade superior às mulheres $\left(1,37 \mathrm{~m} \cdot \mathrm{seg}^{-1}\right.$ e $1,23 \mathrm{~m} \cdot \mathrm{seg}^{-1}$, respectivamente). Uma possível razão para esta diferença entre os sexos pode ser pelo fato de os homens usualmente terem maior ACR, como demonstrado nos recentes estudos conduzidos por Buzzachera et al. ${ }^{(16)}$ e Krinski et al.(17) os quais relataram que indivíduos com maior nível de ACR tendem a intuitivamente selecionar uma velocidade de caminhada superior.

Como consequência da maior velocidade de caminhada autosselecionada, os homens demonstraram maior $\dot{\mathrm{V}}_{2}$ durante os 20 minutos de caminhada em ritmo autosselecionado na esteira comparado com as mulheres. Prévias investigações têm reportado maiores taxas de consumo de oxigênio absoluto em homens durante exercício que em mulheres ${ }^{(6)}$. Controversamente não tem sido reportado diferença entre os sexos ${ }^{(18)}$. Entretanto, nenhum desses estudos examinou estas respostas a um ritmo autosselecionado. Uma investigação que estudou as diferenças do sexo usando modelo multimodal de exercício executada 
em ritmo autosselecionado demonstrou resultados similares aos da presente investigação, revelando que os homens obtiveram consumo de oxigênio significativamente superior ao das mulheres durante os 20 minutos de exercício(19).

Apesar das diferenças entre os gêneros com relação ao $\dot{\mathrm{V}}_{2}$ absoluto, podemos observar que ambos os grupos caminharam em intensidade relativa similar $\left(\% \dot{V} O_{2}\right.$ máx $)$. Estes achados estão de acordo com os encontrados por Green et al. ${ }^{(6)}$, os quais avaliaram 15 homens e 15 mulheres submetidas a dois protocolos de exercício envolvendo cicloergômetro e esteira e verificaram que, embora os homens obtivessem um maior consumo de oxigênio em termos absolutos comparado as mulheres, ambos os sexos exercitavam-se em intensidade relativa similar. Cabe ressaltar que o protocolo utilizado por Green et al. ${ }^{(6)}$ foi realizado em intensidade preestabelecida. Os achados do presente estudo demonstram que, se a comparação entre os gêneros é baseada em uma similar intensidade relativa de exercício $\left(\% \dot{\mathrm{V}} \mathrm{O}_{2 \text { Máx }}\right)$, isto irá refletir em maior intensidade absoluta $\left(\dot{\mathrm{V}} \mathrm{O}_{2}\right)$ para os homens, comparado às mulheres, devido a estes apresentarem maior nível de condicionamento.

Outro achado interessante da presente investigação é que ambos os sexos selecionaram intensidade de caminhada abaixo das diretrizes propostas pelo $\mathrm{ACSM}^{(2)}$, para manutenção e melhora da $\mathrm{ACR}$, que deve estar dentro de uma faixa de 50-85\% para o $\dot{V} O_{2 \text { Máx }}$ e de $55-90 \%$ da $\mathrm{FC}_{\text {Máx }}$. Estes achados são contrários aos verificados por Buzzachera et al. ${ }^{(20)}$ o qual avaliou 41 mulheres sedentárias com idade de 32,6 \pm 8,6 anos, demonstrando que estas buscaram autosselecionar intensidade de caminhada de $57,3 \pm 12,1 \%$ do $V_{2} O_{2 a ́ x}$ e 74,4 $\pm 9,3 \%$ da $F_{\text {Máx. As }}$ diferenças encontradas no presente estudo para a investigação conduzida por Buzzachera et al. ${ }^{(20)}$ pode ser em decorrência das diferenças verificadas na amostra avaliada, a qual foi composta por mulheres sedentárias com menor nível de ACR e idade superior.

No entanto, resultados semelhantes aos do presente estudo foram encontrados em uma recente investigação conduzida por Krinski et al. ${ }^{(17)}$, os quais verificaram que sujeitos jovens fisicamente ativos selecionam intensidade de caminhada abaixo do proposto pelo $\mathrm{ACSM}^{(2)}$, para manutenção e melhora da ACR, revelando que a caminhada demonstra-se como atividade insuficiente para propiciar esforço considerado efetivo para aprimorar a ACR nesta população.

Em relação às respostas perceptuais, o presente estudo demonstrou não haver diferenças estatisticamente significativas entre os sexos $\left(\right.$ RPE 10). Em contraste, O'Connor et al. ${ }^{(7)}$ encontrou maiores valores para PSE em mulheres comparados com homens; entretanto, cabe ressaltar que estes achados foram baseados em exercício com intensidade preestabelecida e realizado em cicloergômetro de braço. Entretanto, Kravitz et al. ${ }^{(19)}$ reportaram não haver diferenças para a PSE entre os sexos durante exercício realizado em esteira, simulador de esqui e cicloergômetro realizados em intensidades autosselecionadas. Estes resultados podem ser explicados pelos achados de Garcin et al. ${ }^{(8)}$, que demonstraram efeito significante do sexo quando um critério absoluto do $\dot{\mathrm{V}}_{2}$ foi utilizado; entretanto, nenhum efeito foi observado para intensidade relativa $\% \dot{\mathrm{V}} \mathrm{O}_{2 \text { Máx. }}$. Estes achados consistentemente sugerem que a PSE apresenta diferença mínima para o critério fisiológico relativo $\left(\% \dot{V} O_{2 \text { Máx }}\right.$ e \%FC Máx $_{1}$; contudo, diferenças são observadas quando o critério absoluto ( $\dot{\mathrm{V}} \mathrm{O}_{2}$ e FC) é estabelecido.

\section{CONCLUSÃO}

Os resultados da presente investigação demonstram, durante a caminhada em intensidade autosselecionada, que os homens buscam autosselecionar velocidade de caminhada estatisticamente superior à das mulheres e, consequentemente, obtiveram um maior $\dot{\mathrm{V}}_{2}$, fator que pode ter sido decorrente da maior ACR encontrada na amostra masculina. Contudo, ambos os gêneros caminharam em similar intensi-

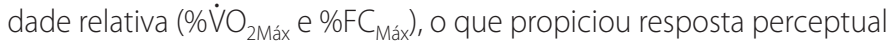
semelhante entre homens e mulheres. Além disso, foi verificado que jovens adultos fisicamente ativos buscam selecionar intensidade de caminhada considerada inferior às diretrizes propostas pelo ACSM(2), necessitando de atividade de intensidade mais vigorosa para manutenção e melhoria da ACR. Os achados da presente investigação devem ser analisados com cautela, visto que a extrapolação destes resultados pode demonstrar diferenças quando aplicados a outras populações (sujeitos com idade superior, sedentários, atletas, pacientes em condições especiais e sujeitos obesos).

\section{AGRADECIMENTOS}

Os autores agradecem as bolsas de estudo cedidas pelo Institute per il Credito Sportivo Italy e a Coordenação de Aperfeiçoamento de Pessoal de Nível Superior, além do apoio recebido pela Cosmed Pulmonary Function Equipament da Itália pelos equipamentos cedidos que permitiram o desenvolvimento do presente estudo.

Todos os autores declararam não haver qualquer potencial conflito de interesses referente a este artigo.

\section{REFERÊNCIAS}

1. Dishman RK, Farqyhar RP, Cureton KJ. Responses to preferred intensity of exercise in men differing in activity level. Med Sci Sports Exerc 1994;26:783-90.

2. American College of Sports Medicine RM. ACSM's guidelines for exercise testing and prescription, seventh ed. Philadelphia: Lippincott Williams and Wilkins, 2006.

3. Da Silva SG, Guidetti L, Buzzachera CF, Elsangedy HM, Colombo H, Krinski K, et al. The influence of adiposity on physiological, perceptual, and affective responses during walking at a self-selected pace. Percept Mot Skills. 2009;109:1-20.

4. Johnson JH, Phipps LK. Preferred method of selecting exercise intensity in adult women. J Strength Cond Res 2006;20:446-9.

5. Robertson RJ, Moyna NM, Sward KL, Millich NB, Goss FL, Thompson PD. Gender comparison of RPE at absolute and relative physiological criteria. Med Sci Sports Exerc 2000;32:2120-9.

6. Green JM, Crews TR, Bosak AM, Peveler WW. Overall and differentiated ratings of perceived exertion at the respiratory compensation threshold: effects of gender and mode. Eur J Appl Physiol 2003;89:445-50.

7. O'Connor PJ, Raglin JS, Morgan WP. Psychometric correlates of perception during arm ergometry in males and females. Int J Sports Med 1996;17:462-6.

8. Garcin M, Fleury A, Mille-Hamard L, Billat V. Sex-related differences in ratings of perceived exertion and estimated time limit. Int J Sports Med 2005;26:675-81.

9. Bruce RA. Exercise testing of patients with coronary artery disease. Ann Clin Res 1971;3:323-32.

10. Gordon CC, Chumlea WC, Roche AF. Stature, recumbent length and weight. In: Lohman TG, Roche AF, Martorell R. Anthropometric standardization reference manual. Champaign: Human Kinetics Books; 1988. p. 3-8.
11. Durnin JV, Womersley J. Body fat assessed from total body density and its estimation from skinfold thickness: measurements on 481 men and women aged from 16 to 72 years. Br J Nutr 1974;32:77-97.

12. Siri WE. Body composition from fluid spaces and density. In: Brozek J, Henschel A. (Eds.). Techniques for measuring body composition. Washington: National Academy of Science, 1961;223-44.

13. Caiozzo VJ, Davis JA, Ellis JF, Azus JL, Vandagriff R, Prietto CA, et al. A comparison of gas exchange indices used to detect the anaerobic threshold. J Appl Physiol 1982;53:1184-9.

14. Cohen J. A power primer. Psychol Bull 1992;112:155-9.

15. Finley FR, Cody KA. Locomotive characteristics of urban pedestrians. Arch Phys Med Rehabil 1970;51:423-6.

16. Buzzachera CF, Elsangedy HM, Colombo H, Krinski K, Coelho RW, Campos W, et al. Effect of Fitness Level on Physiological, Perceptual, and Affective responses during Treadmill Walking at a Self-Selected Pace. Med Sci Sports Exerc 2008;40:5366.

17. Krinski K, Da Silva SG, Elsangedy HM, Colombo H, Buzzachera CF, Santos BV, et al. Respostas fisiológicas durante a caminhada na esteira em ritmo autosselecionado: comparação entre os gêneros. Rev Bras Cineantropom Desempenho Hum 2009;11:307-313.

18. Maffeis C, Schutz Y, Schena F, Zaffanello M, Pinelli L. Energy expenditure during walking and running in obese and nonobese prepubertal children. J Pediatr 1993;123:193-9.

19. Kravitz L, Robergs RA, Heyward VH, Wagner DR, Powers K. Exercise mode and gender comparisons of energy expenditure at self-selected intensities. Med Sci Sports Exerc 1997;29:1028-35.

20. Buzzachera CF, Elsangedy HM, Hallage T, Da Silva SG. Parâmetros fisiológicos e perceptivos durante caminhada de intensidade preferida por mulheres adultas, previamente sedentárias. Rev Bras Cineantropom Desempenho Hum 2007;9:170-6. 